This is the final peer-reviewed accepted manuscript of:

Caselli, F., Sentinelli, P. The generalized lifting property of Bruhat intervals. J Algebr Comb 45, 687-700 (2017).

The final published version is available online at: https://doi.org/10.1007/s10801016-0721-7

Rights / License:

The terms and conditions for the reuse of this version of the manuscript are specified in the publishing policy. For all terms of use and more information see the publisher's website.

This item was downloaded from IRIS Università di Bologna (https://cris.unibo.it/)

When citing, please refer to the published version. 


\title{
THE GENERALIZED LIFTING PROPERTY OF BRUHAT INTERVALS
}

\author{
FABRIZIO CASELLI\# AND PAOLO SENTINELLI *
}

\begin{abstract}
In [E. Tsukerman and L. Williams, Bruhat Interval Polytopes, Advances in Mathematics, 285 (2015), 766-810] it is shown that every Bruhat interval of the symmetric group satisfies the so-called generalized lifting property. In this paper we show that a Coxeter group satisfies this property if and only if it is finite and simply-laced.
\end{abstract}

\section{INTRODUCTION}

The Kostant-Toda lattice is an integrable Hamiltonian system which has been recently studied in detail by Kodama and Williams in [12]; in this paper particular attention is devoted to the asymptotic behaviour of the flow corresponding to an initial point associated with (a given point in) a cell $\mathcal{R}_{u, v}^{+}$of the totally non negative flag variety, where $[u, v]$ is a Bruhat interval in the symmetric group $S_{n}$. When considering a natural multivariable generalisation of this problem, called the full Kostant-Toda hierarchy, they also proved that the moment polytope associated to the flow with initial point corresponding to $\mathcal{R}_{u, v}^{+}$is what they called a Bruhat interval polytope as it can be described as the convex hull in $\mathbb{R}^{n}$ of permutation vectors $(w(1), \ldots, w(n))$ as $w$ varies in the given Bruhat interval.

In [13] Tsukerman and Williams studied some combinatorial aspects of a Bruhat interval polytope and in particular they found a dimension formula and proved that every face of a Bruhat interval polytope is itself a Bruhat interval polytope. The key result that they used in this study is what they called the generalized lifting property which generalizes the (standard) lifting property for symmetric groups and was surprisingly remained unnoticed so far. This generalized lifting property asserts that for every $u<v$ in the symmetric group and any "minimal" reflection (i.e. a transposition) $t$ such that $u<u t$ and $v>v t$, we have $u \triangleleft u t \leqslant v$ and $u \leqslant v t \triangleleft v$, where the symbol $\triangleleft$ denotes the covering relation in Bruhat order. The main fact about this property is that such minimal reflection always exists. The (standard) lifting property of a Bruhat interval is a classical feature of the theory of a Coxeter group $(W, S)$ : it says that if $[u, v]$ is a closed Bruhat interval in $W$ and $s \in S$ are such that $v>v s$ and $u<u s$ then $u \triangleleft u s \leqslant v$ and $u \leqslant v s \triangleleft v$, although it does not ensure that such simple reflection $s$ exists. This property is well-known and is a basic tool in the combinatorics and geometry of Coxeter groups (see, e.g., [11, Chapter 5], [2, Chapter 2], [8]) and has also found important applications in the combinatorics of Kazhdan-Lusztig polynomials (see, e.g., [3,6]).

The main target of this paper is to understand which Coxeter groups satisfy such generalized lifting property; our final result is that a Coxeter group satisfies this property if and only if it is finite and simply-laced. The proof uses in an essential way results of Dyer on the Bruhat graph of a Coxeter group and a geometric representation of an affine Coxeter groups as a group generated by affine reflections in a vector space. The proof makes no use of the

* The second author was supported by MIUR grant FIRB-RBFR12RA9W-002 "Perspectives in Lie theory". 
classification of special families of Coxeter groups except maybe in the observation that an infinite simply-laced Coxeter groups always contains a parabolic subgroup isomorphic to an affine Weyl group.

The paper is organized as follows. In $\S 2$ we establish the notation, we introduce all the preliminary results in the theory of reflection and Coxeter groups that are needed, and we show some general results which link the generalized lifting property to the presentation of a Coxeter group as a reflection group. In $§ 3$ we study some properties of finite simply-laced Coxeter groups which are needed in the proof of the generalized lifting property fro such groups. In $\S 4$ we consider affine Weyl groups and we prove some further basic facts that will allow us to complete the proof of our main result in $\S 5$.

\section{Notation AND PRELIMINARIES}

We begin by establishing some notation. $\mathbb{N}$ is the set of non-negative integers and, if $n \in \mathbb{N}$, $[n]:=\{1,2, \ldots, n\}$; in particular $[0]=\varnothing$. We denote by $|X|$ the cardinality of a set $X$.

Next we recall some basic results in the theory of Coxeter groups and reflection groups which will be useful in the sequel. The reader can consult the fundamental books $[2,4,11]$ as comprehensive sources for this theory and in particular for the undefined notation, results stated without proof, and for further details.

Let $(W, S)$ be a Coxeter system. If $v, w \in W$ we define $\ell(v, w):=\ell(w)-\ell(v)$, where $\ell(z)$ is the length of the element $z \in W$ with respect to $S$. We let

$$
\begin{aligned}
& D_{L}(w):=\{s \in S \mid \ell(s w)<\ell(w)\}, \\
& D_{R}(w):=\{s \in S \mid \ell(w s)<\ell(w)\} .
\end{aligned}
$$

The parabolic subgroup $W_{J} \subseteq W$ is the subgroup with $J \subseteq S$ as generator set. In particular $W_{S}=W$ and $W_{\varnothing}=\{e\}$.

We consider on $W$ the Bruhat order $\leqslant$ (see, e.g., [2, Chapter 2] or [11, Chapter 5]). With $[u, v]$ is denoted an interval in $W$, i.e., if $v, w \in W$,

$$
[v, w]:=\{z \in W \mid v \leqslant z \leqslant w\} .
$$

The relation $v \triangleleft w$ means that $u<v$ and $\ell(u, v)=1$.

We recall the following fundamental property of the Bruhat order, known as the lifting property (see [2, Proposition 2.2.7]):

Proposition 2.1. Let $v, w \in W$ be such that $v<w$ and $s \in D_{R}(w) \backslash D_{R}(v)$. Then $v \leqslant w s$ and $v s \leqslant w$.

A Coxeter system $(W, S)$ is called simply-laced if $m(s, r) \leqslant 3$ for every $s, r \in S, m$ being its Coxeter matrix.

The set $T=\left\{w s w^{-1} \mid s \in S, w \in W\right\}$ is the set of reflections of a Coxeter system $(W, S)$. For a Coxeter group $W$ we let, for any $u, v \in W$,

$$
\begin{aligned}
D(u) & :=\{t \in T \mid u t<u\}, \\
A(u) & :=\{t \in T \mid u<u t\}, \\
A D(u, v) & :=A(u) \cap D(v) .
\end{aligned}
$$

A finite reflection group is a finite subgroup of $G L(V)$, where $V$ is a finite dimensional real vector space, which is generated by reflections, i.e. elements of order 2 that fix a hyperplane 
pointwise. Let $W$ be a finite reflection group and $T$ be the set of all reflections in $W$. For each reflection $t \in T$ we denote by $H_{t}$ the hyperplane fixed by $t$ and call this the reflecting hyperplane associated to the reflection $t$. Moreover, for every reflection $t \in T$ one can choose a non-zero vector $\alpha_{t} \in V$ such that

- $t\left(\alpha_{t}\right)=-\alpha_{t}$ for all $t \in T$;

- the set $\Phi=\left\{ \pm \alpha_{t}: t \in T\right\}$ is invariant under the action of $W$.

The set $\Phi$ is called the set of roots and we assume without lack of generality that $\Phi$ spans $V$. The connected components of the complement of the union of all reflecting hyperplanes are called chambers and we recall that the action of $W$ on the set of chambers is simply transitive. If we fix a chamber $\mathcal{C}_{0}$ (that will be called fundamental chamber) we let $H_{t}^{+}$be the halfspace determined by $H_{t}$ which contains $\mathcal{C}_{0}$ and we let

$$
\Phi^{+}=\Phi^{+}\left(\mathcal{C}_{0}\right)=\bigcup_{t \in T}\left\{ \pm \alpha_{t}\right\} \cap H_{t}^{+} .
$$

Then there exists a unique set of roots $\Delta \subset \Phi^{+}$, called base, such that

- $\Delta$ is a linear basis of $V$;

- every root in $\Phi^{+}$can be expressed as a linear combination with non-negative coefficients of the elements in $\Delta$.

Changing $\alpha_{t}$ with $-\alpha_{t}$ if necessary we can assume that $\Phi^{+}=\left\{\alpha_{t}, t \in T\right\}$. If we let $S \subset T$ be the indexing set of $\Delta$, i.e.

$$
\Delta=\left\{\alpha_{s}: s \in S\right\}
$$

we have that $(W, S)$ is a finite Coxeter system whose set of reflections is exactly $T$, and every finite Coxeter system arises in this way as a finite reflection group. In the sequel we will always assume that a finite Coxeter group comes equipped with the structure of a finite reflection group as above.

The set of reflections $T$ is partially ordered by letting, for all $r, t \in T, r \prec t$ if $\alpha_{t}-\alpha_{r}$ is still a linear combination of the roots $\alpha_{s}, s \in S$, with non-negative coefficients.

The length function of an element $w \in W$ and the set $D(w)$ have the following geometric interpretation which will be fundamental in our study. The length of an element $w \in W$ equals the number of reflecting hyperplanes which separate the fundamental chamber $\mathcal{C}_{0}$ from the chamber $\mathcal{C}_{w}:=w^{-1}\left(\mathcal{C}_{0}\right)$. A direct consequence is that the set $D(w)$ equals the set of reflections $t \in T$ such that the associated reflecting hyperplane separates $\mathcal{C}_{w}$ from $\mathcal{C}_{0}$, and in particular we have

(1) \{lunghezzaw

$$
\ell(w)=|D(w)| .
$$

Remark 2.2. An immediate consequence of (1) is that, if $\ell(u)<\ell(v)$ and in particular if $u<v$, then $|A D(u, v)| \geqslant \ell(u, v)>0$, since $|A D(u, v)|=\ell(u, v)+|D(u) \backslash D(v)|$. In particular, if $|A D(u, v)|=1$ we have $\ell(u, v)=1$.

We will be interested also in the action of reflections on halfspaces determined by the reflecting hyperplanes. It is clear that for any reflection $t$ we have $t\left(H_{r}\right)=H_{r^{t}}$ where $r^{t}:=t r t$ and therefore we have that either $t\left(H_{r}^{+}\right)=H_{r^{t}}^{+}$or $t\left(H_{r}^{+}\right)=H_{r^{t}}^{-}$.

The following result is crucial in our work.

Proposition 2.3. Let $r, t \in T$. The following are equivalent 
(1) $t\left(H_{r}^{+}\right)=H_{r^{t}}^{-}$;

(2) $r \in D(t)$;

(3) for some $u \in W$ we have $r \in D(u)$ if and only if $r^{t} \in A(u t)$.

(4) for every $v \in W$ we have $r \in D(v)$ if and only if $r^{t} \in A(v t)$.

Proof. (1) implies (2). Since $\mathcal{C}_{r} \subset H_{r}^{-}$we have $\mathcal{C}_{r t}=t\left(\mathcal{C}_{r}\right) \subset H_{r^{t}}^{+}$. Therefore $r^{t} \in A(r t)$ i.e. $t>r t$. Since the map $w \mapsto w^{-1}$ is an automorphism of $W$ as a poset, we also have $t>t r$ i.e. $r \in D(t)$.

(2) implies (3). Letting $u=t$ we clearly have $r \in D(u)$ and $r^{t} \in A(e)$.

(3) implies (1). We have either $\mathcal{C}_{u} \in H_{r}^{-}$and $\mathcal{C}_{u t} \in H_{r^{t}}^{+}$or $\mathcal{C}_{u} \in H_{r}^{+}$and $\mathcal{C}_{u t} \in H_{r^{t}}^{-}$. As $\mathcal{C}_{u t}=t\left(\mathcal{C}_{u}\right)$ it follows that $t\left(H_{r}^{-}\right)=H_{r^{t}}^{+}$.

(1) implies (4). Let $v \in W$. We have $r \in D(v)$ if and only if $\mathcal{C}_{v} \in H_{r}^{-}$and $r^{t} \in A(v t)$ if and only if $\mathcal{C}_{v t}=t\left(\mathcal{C}_{v}\right) \in H_{r^{t}}^{+}$and since $t\left(H_{r}^{-}\right)=H_{r^{t}}^{+}$the two conditions are equivalent.

(4) implies (2) Take $v=t$ and the result follows.

Corollary 2.4. Let $r, t \in T, r \neq t$, be such that $r t=t r$. Then $r \in A(t)$.

Proof. By contradiction, if $r \in D(t)$, we have $t\left(H_{r}^{+}\right)=H_{r}^{-}$, by Proposition 2.3. This implies $H_{t} \subseteq H_{r}$ and so $H_{r}=H_{t}$; moreover $r\left(\alpha_{t}\right)$ is still an eigenvector for $t$ of eigenvalue -1 , since $r$ and $t$ commute. Therefore $r\left(\alpha_{t}\right)=c \alpha_{t}$ and since $r$ is reflection we necessarily have $c=-1$ and hence $r=t$.

If $t \in T$, we have the following partition of the set $T$ :

$$
T=\{t\} \sqcup(D(t) \backslash\{t\}) \sqcup A(t),
$$

and an involution $T \rightarrow T$ defined as the conjugation by $t$, i.e.

$$
r^{t}:=t r t
$$

for all $r \in T$.

Lemma 2.5. The sets $\{t\}, D(t) \backslash\{t\}$ and $A(t)$ are invariant under conjugation by $t$, for all $t \in T$.

Proof. Clearly $\{t\}$ is invariant. Let $r \in D(t) \backslash\{t\}$, i.e. $e<r t<t$ or, equivalently $e<t r=$ $(r t)^{-1}<t$, since the inversion is an automorphism of the poset $(W, \leqslant)$; then $e<r^{t} t=t r<t$. It follows that $A(t)$ is invariant too.

The following theorem generalizes, in the symmetric group, the lifting property stated in Proposition 2.1; this result will be extended to the case of finite simply-laced Coxeter groups and this is the main motivation of the paper.

Theorem 2.6. [13, Theorem 3.3] Let $[u, v], u<v$, be a Bruhat interval in the symmetric group $A_{n}$ and $t$ be a minimal element in $A D(u, v)$ with respect to $\prec$. Then $u \triangleleft u t \leqslant v$ and $u \leqslant v t \triangleleft v$.

We end this section recalling some known facts about the $R$-polynomials; see $[2$, Chapter 5], [11, Chapter 7] and the references there for more information. We just recall here that for any Coxeter group $W$ there exists a unique family of polynomials $\left\{R_{u, v}\right\}_{u, v \in W} \subseteq \mathbb{Z}[q]$ such that, for all $u, v \in W$ :

(1) $R_{u, v}=0$ if $u \nless v$; 
(2) $R_{v, v}=1$;

(3) if $u<v$ and $s \in D_{R}(v)$ then

$$
R_{u, v}= \begin{cases}R_{u s, v s}, & \text { if } s \in D_{R}(u), \\ q R_{u s, v s}+(q-1) R_{u, v s}, & \text { otherwise. }\end{cases}
$$

These polynomials are called $R$-polynomials. In the hypothesis of Theorem 2.6 , the simple reflection in the recursion (2) can be replaced by any minimal reflection in $A D(u, v)$.

Proposition 2.7. [13, Proposition 5.3] Let $[u, v], u<v$, be a Bruhat interval in the symmetric group $A_{n}$ and $t$ be a minimal element in $A D(u, v)$. Then

$$
R_{u, v}=q R_{u t, v t}+(q-1) R_{u, v t} .
$$

As shown in [13, Section 5], if $t \in A D(u, v)$ is any reflection such that $u \triangleleft u t \leqslant v$ and $u \leqslant v t \triangleleft v$, the result of the proposition could be not true.

\section{Finite Simply-LACED COXeter GRoups}

A subgroup $W^{\prime}$ of $W$ generated by a subset of $T$ is called a reflection subgroup. The study of reflection subgroups goes back at least to Borel and de Siebenthal [5] and Dynkin [7] (see also $[10, \S 2]$ and the references cited there). We need in particular the following known result.

Proposition 3.1. Let $(W, S)$ be a Coxeter group and $W^{\prime}$ a reflection subgroup and let $S^{\prime}=\left\{t \in W^{\prime} \cap T: D(t) \cap W^{\prime}=\{t\}\right\}$. Then $\left(W^{\prime}, S^{\prime}\right)$ is itself a Coxeter system with Bruhat order induced by the Bruhat order of $(W, S)$ and with set of reflection $T^{\prime}=T \cap W^{\prime}$. If $W$ is finite and simply-laced then $W^{\prime}$ is also finite and simply-laced.

It follows that if $r, t$ are distinct reflections in a finite simply-laced Coxeter group then either $r t=t r$ or $r t r=t r t$. More can be said about the orbits (by right multiplication) of a reflection subgroup by looking at the so-called Bruhat graph. The Bruhat graph of a Coxeter system $(W, S)$ is the directed graph $\Omega_{W, S}$ with vertex set $W$ and edge set

$$
\{(u t, u): u \in W, t \in D(u)\} .
$$

We make the convention to draw an arrow from $x t$ to $x$ and to label it by the reflection $t$ to indicate a directed edge $(x t, x)$. If $A$ is any subset of $W$ we denote by $\Omega_{W, S}(A)$ the directed subgraph of $\Omega_{W, S}$ induced on $A$. The main result that is needed in this paper on the Bruhat graph is the following (see [9, Theorem 1.4]).

Theorem 3.2. Let $(W, S)$ be a Coxeter group and $\left(W^{\prime}, S^{\prime}\right)$ be a reflection subgroup. Then

(1) $\Omega_{W^{\prime}, S^{\prime}}=\Omega_{W, S}\left(W^{\prime}\right)$;

(2) for all $x \in W$ there exists $x_{0} \in x W^{\prime}$ such that the map $W^{\prime} \rightarrow x W^{\prime}$ given by $w \mapsto x_{0} w$ induces an isomorphism of directed graphs between $\Omega_{W^{\prime}, S^{\prime}}$ and $\Omega_{W, S}\left(x W^{\prime}\right)$ that preserve the labels of the edges.

The following is a very special application of Theorem 3.2.

Proposition 3.3. Let $(W, S)$ be a finite simply-laced Coxeter group and $r, t \in T$ with $r \in$ $D(t) \backslash\{t\}$. Then the reflection subgroup $W^{\prime}=\langle r, t\rangle$ has type $A_{2}$, it has Coxeter generators $r, r^{t}$ and in particular $\alpha_{t}=\alpha_{r}+\alpha_{r^{t}}$. 


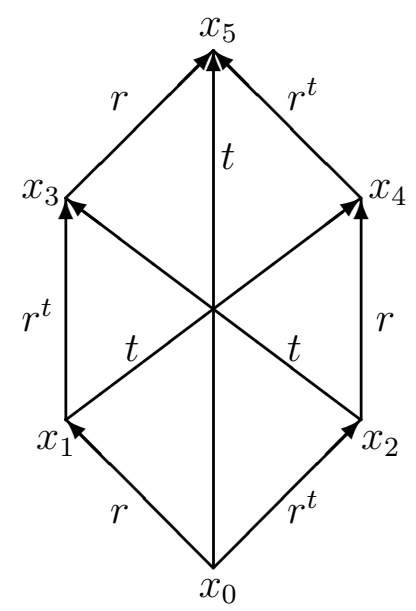

FiguRE 1. Example of induced Bruhat graph.fig:standard

If $x \in W$ then $\Omega_{W, S}\left(x W^{\prime}\right)$ is shown in Figure 1 , where $x$ can be any element in $\left\{x_{0}, x_{1}, x_{2}, x_{3}, x_{4}, x_{5}\right\}$, and where the labeling of the edges is independent of the choice of $x$. In particular we have that $D(x) \cap W^{\prime}$ is one of the following sets:

$$
\emptyset,\{r\},\left\{r^{t}\right\},\{r, t\},\left\{r^{t}, t\right\},\left\{r, r^{t}, t\right\} .
$$

Proof. This result follows directly from Theorem 3.2. The reflections $r, t$ generate a finite simply-laced Coxeter group $W^{\prime}$ of rank 2, i.e. either of type $A_{1} \times A_{1}$ or of type $A_{2}$. Since $r \in D(t)$ we deduce that $r$ and $t$ do not commute by Corollary 2.4 and so $W^{\prime}$ has type $A_{2}$. The reflections in $W^{\prime}$ are $r, t, r^{t}$ and since $r \in D(t)$ we deduce that the Coxeter generators of $W^{\prime}$ are $r$ and $r^{t}$, by Theorem 3.1. The last part of the statement is merely an illustration of Theorem 3.2 in this case.

Corollary 3.4. Let $r, t \in T, r \in D(t) \backslash\{t\}$. Then $r \prec t$.

Proof. By Proposition 3.3 we have $\alpha_{t}=\alpha_{r}+\alpha_{r t}$ and the result follows.

Theorem 3.5. Let $W$ be finite and simply-laced. Let $u, v \in W$ be such that $A D(u, v) \neq \emptyset, t$ be a minimal reflection in $A D(u, v)$ and $r \in D(t) \backslash\{t\}$; then

$$
r \in A(u) \Leftrightarrow r \in A(v) \Leftrightarrow r^{t} \in D(v) \Leftrightarrow r^{t} \in D(u) .
$$

Proof. By Lemma 2.5, Corollary 3.4 and the minimality of $t$ we clearly have that $r \in A(u)$ implies $r \in A(v)$ and that $r^{t} \in D(v)$ implies $r^{t} \in D(u)$.

Let $W^{\prime}=\langle r, t\rangle$. If $r \in A(v)$, since $t \in D(v)$ we necessarily have $D(v) \cap W^{\prime}=\left\{r^{t}, t\right\}$ by Proposition 3.3 and so $r^{t} \in D(v)$.

Similarly, if $r^{t} \in D(u)$, since $t \in A(u)$ we have $D(u) \cap W^{\prime}=\left\{r^{t}\right\}$ by Proposition 3.3 and so $r \in A(u)$.

Proposition 3.6. Let $W$ be finite and simply-laced. Let $u, v \in W$ be such that $A D(u, v) \neq \emptyset$ and $t$ be a minimal reflection in $A D(u, v)$. If $t \notin S$ then there exists $s \in S$ such that 
(1) $s \in D(t)$;

(2) $s t s=t s t$

(3) $s^{t}$ is a minimal element in $A D(u s, v s)$.

Moreover we have $s \in D(v)$ if and only if $s \in D(u)$.

Proof. We let $W^{\prime}=\langle s, t\rangle$ and $s$ be any right descent of $t$.Then condition (1) is trivial and condition (2) follows immediately from Proposition 3.3, applied with $r=s$.

If $s \in D(v)$ and $s \in A(u)$ we contradict the minimality of $t$, since $s \prec t$ by Corollary 3.4.

If $s \in A(v)$ and $s \in D(u)$ we have that $D(u) \cap W^{\prime}=\{s\}$ and $D(v) \cap W^{\prime}=\left\{s^{t}, t\right\}$, by Proposition 3.3. Therefore $s^{t} \in A D(u, v)$ and we contradict again the minimality of $t$, by Lemma 2.5 and Corollary 3.4.

The last statement of the proposition is therefore proved. In order to prove condition (3) we can assume that either $s \in D(v) \cap D(u)$ or $s \in A(v) \cap A(u)$. We assume we are in the former case, the latter being entirely similar.

We refer to Figure 1 for the $W^{\prime}$-orbit of $u$ (with $r=s$ ). Since $D(u)$ contains $s$ and does not contain $t$ we have that $u=x_{1}$ and in particular $s^{t} \in A(u)$ and $s^{t} \in A(u s)$. Since $s^{t} \in A(u)$ we also have $s^{t} \in A(v)$ by the minimality of $t$. Therefore $D(v) \cap W^{\prime}=\{s, t\}$ and if we refer to (another copy of) Figure 1 for the $W^{\prime}$-orbit of $v$ we have $v=x_{4}, v s=x_{2}$ and so $s^{t} \in D(v s)$ concluding that $s^{t} \in A D(u s, v s)$.

It remains to show that $s^{t}$ is minimal in $A D(u s, v s)$ and so we assume by contradiction that there exists $r \prec s^{t}, r \in A D(u s, v s)$. By the standard lifting property we have vsr $<<v$. Now we observe that $u$ and usrs are comparable in Bruhat order since srs is a reflection and so to show that usrs $>u$ it is enough to show that usrs $<u$ does not hold. This again is a direct consequence of the standard lifting property. Therefore we have $\operatorname{srs} \in A D(u, v)$.

Now we observe that $\alpha_{s r s}=\alpha_{r}$ if $s r=r s$ and $\alpha_{s r s}=\alpha_{r} \pm \alpha_{s}$ otherwise. In both cases we have $\alpha_{\text {srs }}=\alpha_{r}+\varepsilon \alpha_{s}$ for some $\varepsilon \in\{0,+1,-1\}$. Therefore

$$
\begin{aligned}
\alpha_{t} & =\alpha_{s}+\alpha_{s^{t}} \\
& \succ \alpha_{s}+\alpha_{r} \\
& =\alpha_{s}+\alpha_{s r s}-\varepsilon \alpha_{s} \\
& \succeq \alpha_{s r s},
\end{aligned}
$$

contradicting the minimality of $t$. The proof is complete.

\section{Affine Weyl groups}

In this section we develop some tools in the theory of affine Weyl groups that will be needed in the study of the generalized lifting property in these groups. An affine Weyl group $\hat{W}$ can be realized in the following way (see [11, Chapter 4] for more details). Let $(W, S)$ be a finite crystallographic Coxeter system acting as a finite reflection group on a vector space $V, T$ its set of reflections and $n:=|S|$; a corresponding affine group is the group of affine transformations generated by (affine) reflections $t^{(k)}$ through all the hyperplanes of the form $H_{t^{(k)}}=\left\{x \in V \mid \phi_{t}(x)=k\right\}$, where $t \in T, k \in \mathbb{Z}$ and $\phi_{t}(x)=0$ is the equation of the hyperplane fixed by $t$. We assume that the linear functions $\phi_{t}$ are chosen in such way that the fundamental chamber $\mathcal{C}_{0}$ of $(W, S)$ sits in the halfspace $H_{t}^{+}:=\left\{x: \phi_{t}(x)>0\right\}$ for all $t \in T$. We also let $\hat{T}:=\left\{t^{(k)}: t \in T, k \in \mathbb{Z}\right\}$ be the set of (affine) reflections of $\hat{W}$. 
Let $\mathcal{H}:=\left\{H_{t^{(k)}} \mid t \in T, k \in \mathbb{Z}\right\}$ and define $H_{t^{(k)}}^{+}:=\left\{x \in \mathbb{R}^{n} \mid \phi_{t}(x)>k\right\}$ and $H_{t^{(k)}}^{-}:=\{x \in$ $\left.\mathbb{R}^{n} \mid \phi_{t}(x)<k\right\}$. The connected components of $\mathbb{R}^{n} \backslash \mathcal{H}$ are called alcoves. The fundamental alcove $\mathcal{A}_{0}$ is obtained by intersecting $\mathcal{C}_{0}$ with the intersection of halfspaces $\bigcap_{t \in T} H_{t^{(1)}}^{-}$; it results that

$$
\mathcal{A}_{0}=\mathcal{C}_{0} \cap H_{t_{0}^{(1)}}^{-}
$$

where $t_{0}$ is the maximum in $(T, \preceq)$ (see [11, Section 4.3]).

The affine Weyl group $\hat{W}$ acts simply transitively on the set of alcoves and therefore we identify an element $w \in \hat{W}$ with the corresponding alcove $\mathcal{A}_{w}:=w^{-1}\left(\mathcal{A}_{0}\right)$.

Moreover, we associate to any pair of alcoves $\mathcal{A}, \mathcal{B}$ an integer

$$
d(\mathcal{A}, \mathcal{B}):=\mid\{H \in \mathcal{H}: H \text { separates } \mathcal{A} \text { and } \mathcal{B}\} \mid .
$$

A sequence of alcoves $\mathcal{A}_{0}, \ldots, \mathcal{A}_{m}$ is called a gallery of length $m$ if for all $i$ we have $d\left(\mathcal{A}_{i}, \mathcal{A}_{i+1}\right)=1$. One has (see [11, Theorem 4.5]):

Proposition 4.1. Let $w \in \hat{W}$. Then

(1) $\ell(w)=d\left(\mathcal{A}_{0}, \mathcal{A}_{w}\right)$;

(2) $\ell(w)=$ the length of the shortest gallery between $\mathcal{A}_{0}$ and $\mathcal{A}_{w}$;

(3) $t^{(k)} \in D(w)$ if and only if the hyperplane $H_{t^{(k)}}$ separates $\mathcal{A}_{0}$ from $\mathcal{A}_{w}$.

If $t \in T$ and $k \in \mathbb{Z}$, let $\operatorname{Str}\left(t^{(k)}\right)$ be the hyperstripe defined by $\operatorname{Str}\left(t^{(k)}\right):=H_{t^{(k-1)}}^{+} \cap H_{t^{(k+1)}}^{-}=$ $\left\{x \in \mathbb{R}^{n}: k-1<\phi_{t}(x)<k+1\right\}$.

Proposition 4.2. Let $\mathcal{A}, \mathcal{B}$ be alcoves such that $\mathcal{A}, \mathcal{B} \not \subset \operatorname{Str}\left(t^{(k)}\right)$. Then

$$
\left|d(\mathcal{A}, \mathcal{B})-d\left(\mathcal{A}, t^{(k)}(\mathcal{B})\right)\right| \geqslant 3
$$

Proof. Without loss of generality we can assume that $\phi_{t}(x)<k-1$ for all $x \in \mathcal{A}$ and $\phi_{t}(y)>k+1$ for all $y \in \mathcal{B}$. Let $\mathcal{A}=\mathcal{A}_{0}, \mathcal{A}_{1}, \ldots, \mathcal{A}_{d}=\mathcal{B}$ be a minimal gallery joining $\mathcal{A}$ to $\mathcal{B}$ and let $i$ be the (unique) index in $[d]$ such that $\phi_{t}(x)<k$ for all $x \in \mathcal{A}_{i}$ and $\phi_{t}(y)>k$ for all $y \in \mathcal{A}_{i+1}$. Then $t^{(k)}\left(\mathcal{A}_{i}\right)=\mathcal{A}_{i+1}$ and therefore

$$
\mathcal{A}=\mathcal{A}_{0}, \mathcal{A}_{1}, \ldots, \mathcal{A}_{i}, t^{(k)}\left(\mathcal{A}_{i+2}\right), \ldots, t^{(k)}\left(\mathcal{A}_{d}\right)=t^{(k)}(\mathcal{B})
$$

is a gallery joining $\mathcal{A}$ and $t^{(k)}(\mathcal{B})$ of length $d-1$. Nevertheless this gallery is not minimal as it crosses the hyperplane $H_{t^{(k-1)}}$ twice, as both $\mathcal{A}$ and $t^{(k)}(\mathcal{B})$ are contained in $H_{t^{(k-1)}}^{-}$while $\mathcal{A}_{i} \in H_{t^{(k-1)}}^{+}$, and the result follows.

The following result will be used to show that in any infinite Coxeter group there is a Bruhat interval for which the generalized lifting property does not hold.

Proposition 4.3. A chamber $\mathcal{C}$ cannot be covered by a finite number of hyperstripes.

Proof. Is is well-known that $\mathbb{R}^{n}$ is not a union of a finite number of hyperplanes and so the same hold for $\mathcal{C}$. So let $x \in \mathcal{C}$ such that $\phi_{t}(x) \neq 0$ for all $t \in T$. As all $\phi_{t}$ are linear it is clear that for every $a \in \mathbb{R}$ there exists $c>0$ such that $\phi_{t}(c x)>a$ for all $t \in T$. As $c x \in \mathcal{C}$ for all $c>0$ the result follows. 


\section{THE GENERALIZED LIFTING PROPERTY}

The lifting property stated in Proposition 2.1 does not ensure that for a Bruhat interval $[u, v]$ there exists a simple reflection $s \in D_{R}(v) \backslash D_{R}(u)$ such that $u \triangleleft u s \leqslant v$ and $u \leqslant v s \triangleleft v$. Of course, if $D_{R}(v) \subseteq D_{R}(u)$, such an $s$ does not exist; in general one can hope that there exists a reflection $t \in A D(u, v)$ for which $u \triangleleft u t \leqslant v$ and $u \leqslant v t \triangleleft v$. This is called the generalized lifting property (GLP) of the interval $[u, v]$ and [13, Theorem 3.3] asserts that in the symmetric groups $A_{n}$ the GLP holds for every interval. Clearly, by Proposition 2.1, an interval of the type $[e, u]$ has the GLP, for every $u>e$, and, in finite groups, the same happens for intervals of the type $\left[u, w_{0}\right]$, for every $u<w_{0}$, where $w_{0}$ denote the unique element of maximal length.

The following example shows that this is not the case in general.

Example 5.1. Let $(W, S)$ be a Coxeter group of rank 2 with $S=\{s, t\}$ and $m_{s, t} \geqslant 4$ (for example the Weyl group of type $\left.B_{2}\right)$. Consider the elements $u=s$ and $v=s t s$. Then $\ell(u, v)=2$ and $[u, v]=\{s, s t, t s, s t s\}$. Therefore $A D(u, v)=\{s t s, s t s t s\}$ but $\ell(u s t s t s)=4>\ell(u)+1$ and $\ell$ (vsts $)=0<\ell(v)-1$.

The example above shows that there is an interval in any Coxeter group which is not simply-laced for which the GLP does not hold.

The following theorems affirm that every interval in a finite simply-laced Coxeter groups, i.e. a direct product of Weyl groups of type $A_{n}, D_{n}, E_{6}, E_{7}$ and $E_{8}$, has the GLP.

Theorem 5.2 (Covering property). Let $W$ be a finite simply-laced Coxeter system, $u, v \in W$ be such that $A D(u, v) \neq \emptyset$ and $t$ be a minimal element in $A D(u, v)$. Then $u \triangleleft u t$ and $v \triangleright v t$.

Proof. To show that $u \triangleleft u t$ we have to prove that $\ell(u)=\ell(u t)-1$ and for this it is enough to show that there exists a bijection $\phi: D(u) \rightarrow D(u t) \backslash\{t\}$. The bijection $\phi$ is the restriction to $D(u)$ of the following involution on $T$ : we let

$$
\phi(r)= \begin{cases}r^{t}, & \text { if } r \in A(t), \\ r, & \text { otherwise }\end{cases}
$$

for all $r \in T$.

The map $\phi$ is a bijection on $T$ by Lemma 2.5 and so to conclude we have to prove that if $r \neq t$ then

(3) $\{$ phibij\}

$$
r \in D(u) \Leftrightarrow \phi(r) \in D(u t) .
$$

First assume $r \in D(t)$, and so also $r^{t} \in D(t)$ by Lemma 2.5. By Proposition 2.3 applied to $r^{t}$ we have $r^{t} \in D(u)$ if and only if $r \in A(u t)$. By Theorem 3.5 we also have $r^{t} \in D(u)$ if and only if $r \in A(u)$ and therefore Eq. (3) follows in this case.

If $r \in A(t)$ all the statements of Proposition 2.3 are false and in particular we have that (3) is implied by the negation of condition (3) in Proposition 2.3.

The result for $v$ follows similarly by observing that $\phi$ restricts to a bijection

$$
\phi: D(v) \backslash\{t\} \rightarrow D(v t) .
$$

We note that the covering property (Theorem 5.2) does not require that $u \leqslant v$. 
Theorem 5.3 (Generalized lifting property). Let $(W, S)$ be a finite simply-laced Coxeter system, $u, v \in W, u<v$ and $t$ be a minimal element in $A D(u, v)$. Then $u \triangleleft u t \leqslant v$ and $u \leqslant v t \triangleleft v$.

Proof. By Theorem 5.2 we only have to show that $u t \leqslant v$ and $u \leqslant v t$. We proceed by induction on the partial order $\prec$ on reflections. If $t$ is minimal in $T$ then $t \in S$ and the result follows by Proposition 2.1. Otherwise choose $s \in S$ as in Proposition 3.6. Since $s^{t} \in D(t)$ we have $s^{t} \prec t$ by Corollary 3.4 and we can apply our induction hypothesis to the pair $(u s, v s)$. It follows that $u s s^{t}<v s$ and. since $s t s=t s t$ by Proposition 3.6, we have $u t s<v s$. To deduce that $u t \leqslant v$ it is enough to prove that $s \in D(u t)$ if and only if $s \in D(v)$. But $s \in D(t)$ and so $s \in D(u t)$ if and only if $s^{t} \in A(u)$ by Proposition 2.3 and hence the result follows from Theorem 3.5. The proof of $u<v t$ is similar.

The next proposition generalizes the result stated in Proposition 2.7.

Proposition 5.4. Let $[u, v]$ be a Bruhat interval in a finite simply-laced Coxeter group and $t$ a minimal element in $A D(u, v)$. Then

$$
R_{u, v}=q R_{u t, v t}+(q-1) R_{u, v t} .
$$

Proof. We prove the result by induction on the rank function of $(T, \preceq)$. If $t \in S$ the result is the recursion (2). Otherwise, choose $s \in S$ as in Proposition 3.6. Then, since $s \in D_{R}(v)$ if and only if $s \in D_{R}(u)$, we have $R_{u, v}=R_{u s, v s}=q R_{u s t s t, v s t s t}+(q-1) R_{u s, v s t s t}=q R_{u t s, v t s}+(q-1) R_{u s, v t s}=$ $q R_{u t, v t}+(q-1) R_{u, v t}$. The last equality follows from the fact that $v<v s$ if and only if $v t<v t s$ if and only if $u<u s$ if and only if ut <uts (for this one can still make use of Figure 1 with the observation that $v t \triangleleft v$ and $u t \triangleright u$ ).

The following example shows that the GLP does not hold in general for infinite simply-laced Coxeter system.

Example 5.5. Let $n \geqslant 3$ and consider the affine Weyl group $\tilde{A}_{n}$, with set of Coxeter generators $S=\left\{s_{1}, s_{2}, \ldots, s_{n}\right\}$ (see [2, Section 8.3] for a combinatorial description). Let $v=s_{1} s_{2} \cdots s_{n-1} s_{n} s_{1} s_{2} \cdots s_{n-1}$ and $u=s_{1} s_{2} \ldots s_{n-1}$. In the combinarial description of $u$ and $v$ as permutations of $\mathbb{Z}$ we have $v=[v(1), \ldots, v(n)]=[3,4, \ldots, n, n+2,-n+1]$ and $u=[u(1), \ldots, u(n)]=[2,3, \ldots, n, 1]$. Letting $t_{i}=u^{-1} s_{n} \cdots s_{i+1} s_{i} s_{i+1} \cdots s_{n} u$ for all $i \in[n]$ one can check that $A D(u, v)=\left\{t_{1}, t_{2}, \ldots, t_{n}\right\}$. Nevertheless, using the combinatorial interpretation of the length function in $\tilde{A}_{n}$ given in [2, Section 8.3] one can conclude that none of the elements $t_{1}, \ldots, t_{n}$ satisfy the covering property for $[u, v]$ (in particular we have $\ell\left(u t_{i}\right)>\ell(u)+1$ for all $i=1, \ldots, n-1$ and $\left.\ell\left(v t_{n}\right)<\ell(v)-1\right)$.

For an infinite simply-laced Coxeter system $(W, S)$, if $s \in S$, the following proposition shows that any interval of the type $[s, u]$ satisfies the GLP.

Proposition 5.6. Let $(W, S)$ be a simply-laced Coxeter system and $s \in S$. Then the GLP holds for the interval $[s, u]$, for every $u>s$.

Proof. If $D_{R}(u) \neq\{s\}$ the result follows by Proposition 2.1. Let $D_{R}(u)=\{s\}$; then, since $(W, S)$ is simply-laced, we have that $s_{1} \ldots s_{k-1} s_{k} s$ is a reduced expression for $u$ and $s \notin D_{R}\left(u s s_{k}\right)$ 
because, otherwise, $s_{k} \in D_{R}(u)$. Then the reflection $t=s s_{k} s$ satisfies $s \triangleleft s t \leqslant u$ and $s \leqslant u t \triangleleft u$.

Our last goal is to show that, in general, Bruhat intervals in infinite Coxeter groups do not satisfy the GLP. Since every infinite simply-laced Coxeter group has a parabolic subgroup isomorphic to an affine Weyl group it is enough to consider this class of groups.

The next lemma states that any element in an affine Weyl group is smaller in the Bruhat order than every element of large enough length.

Lemma 5.7. Let $(W, S)$ be an affine Coxeter system. Then for any $u \in W$ there exists $n \in \mathbb{N}$ such that $u<v$ for all $v \in W$ whose length satisfies $\ell(v)>n$.

Proof. Since every parabolic subgroup $W_{J}$ of $W$ is finite, we let $k$ be the maximum length of an element in a proper parabolic subgroup of $W$. If $u \in W$ and $s_{1} s_{2} \ldots s_{h}$ is a reduced expression for $u$, consider the number $n=h(k+1)$. If $v \in W$ is any element whose length satisfies $\ell(v)>n$, then $v$ should has a reduced expression such as $v_{1} v_{2} v_{3} \ldots v_{h}$, with $\ell\left(v_{i}\right)>k$ for all $i$. In particular $v_{i}$ is not contained in any parabolic subgroups of $W$ and hence $s_{i}<v_{i}$ for all $i \in[h]$ and so $u<v$.

Theorem 5.8. Let $(\hat{W}, S)$ be an affine Coxeter system and $\mathcal{A}_{u}$ be any alcove contained in the chamber $-\mathcal{C}_{0}$. Then there exists $v \in \hat{W}$ such that $u<v$ but the interval $[u, v]$ does not satisfy the covering property.

Proof. Let $W$ and $T$ be as in $\S 4$. The fundamental alcove is given by the inequalities $0<\phi_{t}(x)<1$ for all $t \in T$. If $\mathcal{A}=\mathcal{A}_{u}$ is an alcove in $-\mathcal{C}_{0}$, then it satisfies $\mathcal{A} \in H_{t}^{-}$ for every $t \in T$; therefore $T \subseteq D(u)$ and $\left\{t^{(1)}: t \in T\right\} \subseteq A(u)$. Consider the finite set $R(u)=\left\{t^{(k)} \in \hat{T}: u \triangleleft u t^{(k)}\right\}$; by Proposition 4.3, we can find an alcove $\mathcal{A}_{v} \in-\mathcal{C}_{0}$ such that

$$
\mathcal{A}_{v} \not \subset \bigcup_{t^{(k)} \in R(u)} \operatorname{Str}\left(t^{(k)}\right) \text {. }
$$

Moreover, such element $v$ can be chosen of arbitrary high length (as $-\mathcal{C}_{0} \backslash \bigcup_{t^{(k)} \in R(u)} \operatorname{Str}\left(t^{(k)}\right.$ is unbounded), and in particular we can assume that $u<v$ by Lemma 5.7.

Now let $t^{(k)} \in R(u)$. It is enough to show that $v$ does not cover $v t^{(k)}$. We already know that $k \neq 0$ by construction (since $t=t^{(0)} \in D(u)$ ) and that $t^{(1)} \in A(v)$ by our previous remark. For $k \neq 0,1$ we have that the fundamental alcove $\mathcal{A}_{0}$ is not contained in the hyperstripe $\operatorname{Str}\left(t^{(k)}\right)$ and so we can apply Proposition 4.2 to the pair $\mathcal{A}_{0}$ and $\mathcal{A}_{v}$. In particular we have

$$
\left|\ell(v)-\ell\left(v t^{(k)}\right)\right|=\left|d\left(\mathcal{A}_{0}, \mathcal{A}_{v}\right)-d\left(\mathcal{A}_{0}, t^{(k)}\left(\mathcal{A}_{v}\right)\right)\right| \geqslant 3
$$

and the proof is complete.

By the theorem above, in every infinite Coxeter group there exists an interval which does not satisfy the GLP. Therefore with Example 5.1, Theorem 5.3 and Theorem 5.8 we have proved the following theorem.

Theorem 5.9. Let $(W, S)$ be a Coxeter system. The GLP holds for every Bruhat interval $[u, v]$ in $W$ if and only if $(W, S)$ is finite and simply-laced. 


\section{REFERENCES}

[1] L. Billera and F. Brenti, Quasisymmetric functions and Kazhdan-Lusztig polynomials, Israel J. Math. 184 (2011), 317-348

[2] A. Björner and F. Brenti, Combinatorics of Coxeter Groups, Springer, 2005.

[3] F. Brenti, F. Caselli, M. Marietti, Special matchings and Kazhdan-Lusztig polynomials, Advances in Mathematics 202 (2006), 555-601.

[4] K. Brown, Buildings, Springer, 1989.

[5] A. Borel and J. De Siebenthal, Les sous-groupes fermés de rang maximum des groupes de Lie clos, Comment. Math. Helv., 23:200-221, 1949.

[6] E. Delanoy, Combinatorial invariance of Kazhdan-Lusztig polynomials on intervals starting from the identity, J. Algebraic Combin. 24 (2006), 437-463.

[7] E. B. Dynkin, Semisimple subalgebras of semisimple Lie algebras Amer. Math. Soc. Trans. (2) 6, 111-244, 1957.

[8] F. du Cloux, An abstract model for Bruhat intervals, European J. Combin. 21 (2000), 197-222

[9] M.J. Dyer, On the "Bruhat graph" of a Coxeter system, Comp. Math. 78 (1991), 185-191.

[10] M.J. Dyer and G.I. Lehrer, Reflection subgroups of finite and affine Weyl groups, Trans. Amer. Math. Soc. 363 (2011), 5971-6005.

[11] J. E. Humphreys, Reflection Groups and Coxeter Groups, Cambridge Studies in Advanced Mathematics, 1990.

[12] Y. Kodama and L. Williams The full Kostant-Toda hierarchy on the positive flag variety, Comm. Math. Phys. 335 (2015), 247-283. Comm. in Math. Ph.

[13] E. Tsukerman and L. Williams, Bruhat Interval Polytopes, Advances in Mathematics, 285 (2015), 766-810.

\# Dipartimento di matematica, Università di Bologna, Piazza di Porta San Donato 5, 40126 BOLOGNA, ITALY

*Departamento de Matemáticas, Universidad de Chile, las Palmeras 3425, Nunoa, Santiago, CHILE

E-mail address: \# fabrizio.caselli@unibo.it

E-mail address: * paolosentinelli@gmail.com 